\title{
EDITORIAL
}

\section{Why do you want to publish this paper?}

Since taking over as Chairman of the Editorial Board, I have had the opportunity of reading a large number of typescripts over a very short period of time and the range of styles of presentation has been very surprising. The paper that started me thinking about the topic of this editorial was, however, sent to another journal. The paper was very sound, the experimental procedures clearly described and the statistical analyses appropriate, and yet I was left wondering why the paper had been submitted for publication at all.

A journal such as the British Journal of Nutrition exists to publish and disseminate 'reports of original research or original interpretations of existing knowledge' with an additional requirement that the work should develop nutritional concepts. Clearly the most obvious reason why authors submit papers for publication is that they have, or are convinced that they have, original findings to describe to their scientific peers and anyone else who is interested enough to read the paper. It surprises me that so many authors who have original work or findings to communicate, and who, one imagines, are excited and elated by their results, hide their lights under several bushels of words. Their peers will be able, and prepared, to sort out the pearls of research, but often the average reader will pass the paper by. This is not merely a question of clarity of style but also of the effort made in identifying and conveying the excitement of the original research. Remember that the average reader will only read your paper once, if at all, unless you grasp his attention and show what important new finding you have made. I am not suggesting that 'hyped up' abstracts or conclusions will be welcome or acceptable, but that the importance of new findings be clearly identified.

Many papers that are submitted appear to be, and read like, reports that simply describe work that is original in the sense that it has not been done before, but does not present any original findings or interpretations that develop nutritional concepts. One reads the paper and gains the impression that the authors have done the experiments and see publication as the final stage of the work. The maxim that 'if work is not reported in some way it might as well not have been done' was firmly engrained in my early training and is, I think, sound. However, these types of paper make me wonder about the original case that was made for the work. It requires so much effort and planning to get approval and funding before research can be started that these 'report' papers should be rare. Is it the case that the planned work was full of promise but somehow failed to deliver the new insights that were anticipated?

I accept that observational studies have their place in the literature because they provide the base material from which new science develops. At the same time, the choice of observations and measurements that are made in any study have some thinking behind them; usually that the measurements will produce new insights or test some hypothesis. Why are authors so unwilling to share their hypotheses with their readers? Possibly because there is no tradition of theoretical nutrition where the originator of an hypothesis gets as much credit as the experimenter who conducts the crucial experimental tests of the hypothesis. Describing an hypothesis in itself is not sufficient, the hypothesis must be a plausible one that is in accord with existing knowledge and capable of logical development. 
These 'report' style papers are, of course, the seed corn for continued research funding and this is a powerful motivation and is, one suspects, a major factor in the growth of the scientific literature.

The use of bibliometric criteria for the assessment, promotion and appointment of research workers is of course another pressure for publication. I am told that it is frequently possible to see a rise in the rate of publication as some kind of appraisal approaches; possibly the authors are seeking to influence the cruder bibliometric indicators of their scientific performance; it is difficult to imagine a change in the rate of generation of original ideas. A more charitable view would be that the increase in rate is the reason for the subsequent promotion or favourable review.

The editorial board and referees play a key role in the generation of the bibliometric indices of scientific activity, and thus an unseen role in the professional assessment procedures that use these indices. Refereed papers are given greater weight than reviews or monographs, and the authors of refereed papers have, the administrators argue, been judged by their peers; not, I suggest as publications per se, but as publications that make original contributions to knowledge, either in the discovery of new findings or by providing new insights from existing work. It seems important that the primary reason for publishing in the scientific literature, the dissemination of the results of original research, is protected so that these peer reviews are not down-graded by the acceptance of papers that do not meet this criterion. 